JOURNAL OF THE

AMERICAN MATHEMATICAL SOCIETY

Volume 10, Number 3, July 1997, Pages 553-563

S 0894-0347(97)00236-1

\title{
ESSENTIAL CLOSED SURFACES IN BOUNDED 3-MANIFOLDS
}

\author{
D. COOPER, D. D. LONG, AND A. W. REID
}

\section{INTRODUCTION}

A question dating back to Waldhausen [10] and discussed in various contexts by Thurston (see [9]) is the problem of the extent to which irreducible 3-manifolds with infinite fundamental group must contain surface groups. To state our results precisely, it is convenient to make the definition that a map $i: S \leftrightarrow M$ of a closed, orientable connected surface $S$ is essential if it is injective at the level of fundamental groups and the group $i_{*} \pi_{1}(S)$ cannot be conjugated into a subgroup $\pi_{1}\left(\partial_{0} M\right)$ of $\pi_{1}(M)$, where $\partial_{0} M$ is a component of $\partial M$. This latter condition is equivalent to the statement that the image of the surface $S$ cannot be freely homotoped into $\partial M$.

One of the main results of this paper is the following:

Theorem 1.1. Let $M$ be a compact, connected 3-manifold with nonempty incompressible boundary. Suppose that the interior of $M$ has a complete hyperbolic structure. Then either $M$ is covered by a product $F \times I$, where $F$ is a closed orientable surface, or $M$ contains an essential surface $S$ of genus at least 2.

Furthermore $S$ can be lifted to an embedded nonseparating surface in a finite cover of $M$.

In particular, this gives a complete resolution to Waldhausen and Thurston's questions in the context of manifolds with nonempty incompressible boundary:

Corollary 1.2. Let $M$ be a compact, connected irreducible 3-manifold with nonempty incompressible boundary. Then either $M$ is covered by a product $F \times I$, or $M$ contains an essential closed surface.

In particular, the case that all the boundary components of $M$ are tori gives a generalisation of Corollaries $1 \& 2$ of [2].

Indeed our methods show much more is true. In the course of the proof of Theorem 1.1, in the case when $M$ is hyperbolic, we exhibit a finite cover of $M$ containing a pair of disjoint nonseparating embedded essential closed surfaces. Combining this with some 3-manifold topology shows:

Theorem 1.3. Let $M$ be a compact, connected irreducible 3-manifold with nonempty incompressible boundary. Then either $\partial M$ consists only of tori and is covered by a product $T^{2} \times I$, or $\pi_{1}(M)$ virtually maps onto a free group of rank 2 .

Received by the editors September 5, 1996.

1991 Mathematics Subject Classification. Primary 57M50.

The first two authors were partially supported by the NSF, and the third by the Royal Society.

(c)1997 American Mathematical Society 
The method of proof used in the last clause of Theorem 1.1 has a further application. Defining the peripheral homology to be the image of the map

$$
\text { incl }_{*}: H_{2}(\partial M ; \mathbf{Z}) \longrightarrow H_{2}(M ; \mathbf{Z}),
$$

a stronger version of Waldhausen's question is to ask if one can create nonperipheral homology by passing to a finite sheeted covering. This question was also raised by Gabai in [3]. The proof of Theorem 1.3 creates this homology:

Corollary 1.4. Let $M$ be a compact, connected 3-manifold with nonempty incompressible boundary. Suppose that the interior of $M$ has a complete hyperbolic structure. Then given any integer $K$, either $M$ is covered by a product $F^{2} \times I$, or there is a finite sheeted covering $\tilde{M} \longrightarrow M$ so that $H_{2}(\tilde{M}) /$ incl $_{*}\left(H_{2}(\partial \tilde{M})\right)$ has rank at least $K$.

This paper is organised as follows. Since it contains the main ideas and is perhaps of most interest, $\S 2$ is devoted to the proofs in the case that all the boundary components are incompressible tori. In $\S 3$ we prove a technical lemma which enables the proof in the case of higher genus boundary components.

The results of this paper grew out of attempts to generalise Corollaries $1 \& 2$ of [2]. In particular, the idea of looking at the closed surface obtained by tubing together two parallel copies of a bounded surface using a long annulus and then compressing is contained in [2]. The authors gratefully acknowledge the inspiration provided by this seminal paper.

\section{Proof of the CASE that all boundary Components ARe tori}

Throughout the following, since we may always pass to an orientable cover, we shall assume that all manifolds are orientable. All homology groups have Zcoefficients.

Recall that if $M$ is a complete orientable hyperbolic 3-manifold of finite volume, and noncompact, then the ends of the manifold are all of the form $T^{2} \times[0, \infty)$.

Lemma 2.1. Let $M$ be a 3-manifold with nonempty incompressible boundary, all of whose components are tori. Suppose that the interior of $M$ has a complete hyperbolic structure with finite volume. Then there is a finite cover of $M$ which has at least 3 torus boundary components.

Proof. Fix some torus $T$ in $\partial M$. Choose an element $x$ not in $\pi_{1} T$, and set $y=\left[x^{2}, m\right]$ where $m$ is a nontrivial element of $\pi_{1} T$. Since $M$ is hyperbolic, $y$ is nontrivial.

Moreover, since $\pi_{1} M$ is residually finite, there is a finite group $G$ and an epimorphism $\theta$ of $\pi_{1} M$ onto $G$ with $\theta(y)$ nontrivial. Then the finite covering $M_{\theta}$ which corresponds to the kernel of the homomorphism has at least 3 torus components covering $T$. To see this, note that the index of $H=\theta\left(\pi_{1} T\right)$ in $G$ equals the number of pre-images of $T$ in $M_{\theta}$.

However, if $H$ has index at most 2, then since $H$ is normal in $G, \theta(x)^{2}$ would lie in $H$, an abelian group, so that $\left[\theta\left(x^{2}\right), \theta(m)\right]=\theta\left(\left[x^{2}, m\right]\right)=\theta(y)$ would be trivial, a contradiction.

Definition. An oriented surface $F$ which is properly embedded in a 3-manifold $M$ is said to meet the boundary efficiently if for each component $A$ of $\partial M$ the following condition is satisfied. Either $F \cap A=\emptyset$ or $F \cap A \neq \emptyset$, and giving the boundary components of $F$ the induced orientation, every nonempty subset of components of $F \cap A$ is nonzero in $H_{1}(A)$. 
In this section, we are primarily interested in the case that $\partial M$ consists of tori, in which case the condition may be stated as: all the components of $\partial F$ are parallel circles, oriented the same way, and not bounding a disc in $A$.

Lemma 2.2. Suppose $M$ is a compact orientable 3-manifold with toroidal boundary having at least 3 torus boundary components. Then there is a connected, nonseparating properly embedded incompressible surface $F$ in $M$ which is disjoint from at least one torus boundary component. Furthermore $[\partial F]$ is not zero in $H_{1}(\partial M)$ and $F$ meets the boundary of $M$ efficiently.

Proof. Let $T$ be a torus boundary component of $M$ and $\partial_{-} M=\partial M-T$. Then from the long exact sequence of the pair $(M, \partial M)$ and Poincaré-Lefschetz duality, the rank of

is at least

$$
K \equiv \operatorname{ker}\left[\text { incl }_{*}: H_{1}\left(\partial_{-} M\right) \longrightarrow H_{1}(M)\right]
$$

$$
\frac{\beta_{1}(\partial M)}{2}-2>0
$$

Let $\alpha$ be a primitive class in $K$; then there is a nonseparating properly embedded surface $F$ in $M$ with

$$
[\partial F]=\alpha .
$$

We now arrange that $F$ meets the boundary of $M$ efficiently. First, any components of $\partial F$ which bound discs in $\partial M$ can be capped off. After this, whenever $F \cap \partial M$ contains a pair of curves which are adjacent and parallel but are oppositely oriented on some component of $\partial M$, we can cap them off with an annulus. This surface is therefore disjoint from $T$, and meets the boundary efficiently. Now compress $F$ to obtain an incompressible surface. The resulting surface represents the same relative homology class and so there is a component with boundary nonzero in $H_{1}(\partial M)$.

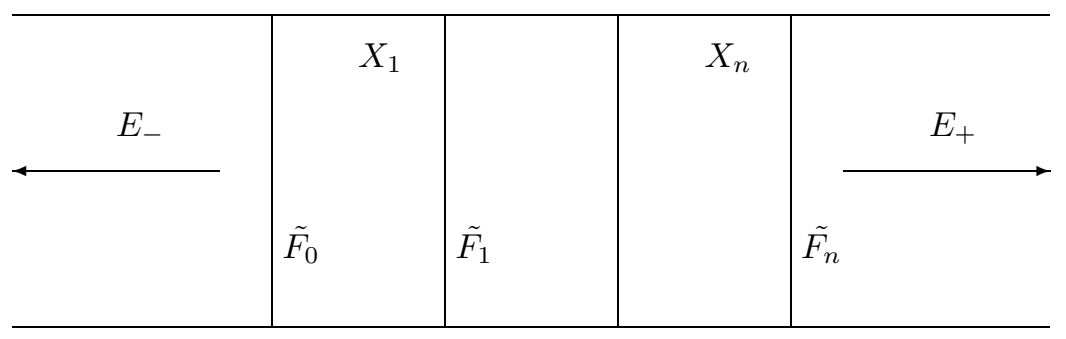

FIGURE 1. The covering $M_{F}$

Theorem 2.3. Let $M$ be a compact, connected 3-manifold with nonempty incompressible boundary, all of whose components are tori. Suppose that the interior of $M$ has a complete hyperbolic structure with finite volume. Then $M$ contains an immersed essential surface $S$ of genus at least 2 .

Furthermore $S$ can be lifted to an embedded nonseparating surface in a finite cover of $M$.

Proof. By Lemma 2.1, after passing to a finite cover, we may suppose that the boundary of $M$ contains at least 3 tori.

Now it follows from Lemma 2.2 that we can find $F$, a 2 -sided nonseparating incompressible surface properly embedded in $M$, with $[\partial F]$ nonzero in $H_{1}(\partial M)$. We may also suppose that $F$ meets the boundary of $M$ efficiently, and is disjoint from some torus boundary component of $M$. 
Set $X \cong M-\operatorname{int} N(F)$ and let $X_{i}=X \times i$ be a copy of $X$. Then the infinite cyclic cover

$$
p: M_{F} \longrightarrow M
$$

dual to $F$ is $M_{F}=\bigcup_{i} X_{i}$. Define a submanifold $Y_{n}=\bigcup_{i=1}^{n} X_{i}$. Let $\tilde{F}_{i}$ be the components of $p^{-1} F$ labelled so that $\tilde{F}_{i}=X_{i} \cap X_{i+1}$. Since $F$ is incompressible in $M$ it follows that $\tilde{F}_{i}$ is incompressible in $M_{F}$. Then

$$
M_{F}=E_{-} \cup_{\tilde{F}_{0}} Y_{n} \cup_{\tilde{F}_{n}} E_{+}
$$

where $E_{-}=\bigcup_{i<0} X_{i}$ and $E_{+}=\bigcup_{i>n} X_{i}$. Two applications of Van Kampen's theorem then implies that

$$
\text { incl }_{*}: \pi_{1} Y_{n} \longrightarrow \pi_{1} M_{F}
$$

is injective, and composing with the covering space projection one sees that

$$
\left(p \mid Y_{n}\right)_{*}: \pi_{1} Y_{n} \longrightarrow \pi_{1} M
$$

is injective.

Partition the boundary of $Y_{n}$ into two parts: $G$ is the component containing $\tilde{F}_{0} \cup \tilde{F}_{n}$ and is diffeomorphic to the double of $F$; and $W$ is the rest of $\partial Y_{n}$.

Let $S$ be the possibly disconnected surface in $Y_{n}$ obtained by compressing $G$ in $Y_{n}$ as much as possible. Since $[G]+[W]=0$ in $H_{2}\left(Y_{n}\right)$ it follows that $[S]=-[W]$. Suppose that some component of $S$ is a sphere; then since $M$ is hyperbolic, $S$ bounds a ball $B$ in $M_{F}$. Now $E_{-}, E_{+}$are both noncompact, thus $B$ is contained in $Y_{n}$. Let $S^{\prime}$ be the subsurface of $S$ obtained by deleting the sphere components. Then $\left[S^{\prime}\right]=-[W]$ also. Suppose that every component of $S^{\prime}$ is parallel to some component of $W$. The only relation between the classes in $H_{2}\left(Y_{n}\right)$ represented by the boundary components of $Y_{n}$ is that their sum is zero. Thus the components of $W$ (which excludes $G$ ) freely generate a subgroup of $H_{2}\left(Y_{n}\right)$. The equation $\left[S^{\prime}\right]=-[W]$ thus implies that $S^{\prime}$ has at least as many components as $[W]$.

Now suppose that we have chosen $n$ so that $n>\beta_{1}(F)$. We claim that this implies that some component $S_{1}$ of $S^{\prime}$ is not parallel to any component of $W$.

Suppose to the contrary that every component of $S^{\prime}$ is parallel to some boundary component of $W$ in $Y_{n}$. If we let $D$ be any boundary component of $\partial M$ which is disjoint from $F$, then $W$ has at least $n$ such components since each component of $D$ lifts. However, each component of $\partial M$ is a torus and so the observations of the previous paragraph show that we have the inequality: $\beta_{1}\left(S^{\prime}\right) \geq 2 n$. But

$$
\beta_{1}\left(S^{\prime}\right) \leq \beta_{1}(G)=2 \beta_{1}(F) .
$$

We see that this is a contradiction with our chosen $n$, so that we have shown that some component $S_{1}$ of $S^{\prime}$ is an embedded incompressible surface in $Y_{n}$ not parallel to any component of $W$.

We must now show that the projection of the surface $S_{1}$ is not homotopic into the boundary of $M$. Suppose to the contrary that it were. Notice that this boundary component cannot be any of the boundary components which meet $F$. The reason is that $F$ meets the boundary of $M$ efficiently, so every such component in $\partial M$ which meets $F$ is covered by noncompact surfaces, in particular there would be free homotopy classes on $S_{1}$ which did not lift, a contradiction.

Then we lift this homotopy back into $M_{F}$ and see a homotopy between an embedded surface $S_{1}$ and some component $\partial_{W} M$ of the boundary of $M_{F}$ which is a pre-image of a boundary component not meeting $F$. Two disjoint embedded 
surfaces in a 3-manifold which are homologous cobound an embedded submanifold $H$.

We have already argued that $\partial_{W} M$ cannot lie in $Y_{n}$. However, this means that $G$ separates the boundary components of $H$, so that $G$ lies in the interior of $H$. Since $G$ meets $\partial M_{F}$ along some annuli, the interior of $H$ meets these annuli in $\partial M_{F}$ which is absurd. This contradiction shows that the projection of $S_{1}$ cannot be homotoped into $\partial M$.

Notice that it follows from the construction that the surface $S_{1}$ embeds in a finite cyclic covering of a finite covering of the manifold $M$.

Corollary 2.4. Let $M$ be an irreducible compact, connected 3-manifold with nonempty boundary, all of whose components are incompressible tori. Then either $M$ is covered by $T^{2} \times I$, or $M$ contains an essential surface.

Proof. The manifold $M$ is Haken, so it has a torus decomposition; if this decomposition contains an essential torus, then we are finished.

So we may assume that all tori in the torus decomposition are boundary parallel. It follows from Thurston's geometrization of Haken manifolds that $M$ is either Seifert fibred or hyperbolic.

If the manifold is Seifert fibred we consider possibilities for the base. This must have boundary, so that it must be a flat or hyperbolic 2-orbifold. One sees easily (for example by taking a manifold covering) that if the base is hyperbolic, one can always find a vertical immersed torus satisfying the conclusion of the corollary. Thus the only possibility for failure is if the base is a flat orbifold with boundary which is therefore covered by an annulus. Pulling back gives the case of a virtual product.

The hyperbolic case is covered by Theorem 2.3 .

A particular case of interest of Corollary 2.4 has been the case of a knot exterior in $S^{3}$. We record it separately:

Corollary 2.5. Let $K$ be a nontrivial knot in $S^{3}$. Then $S^{3} \backslash K$ contains a closed essential surface.

We now aim to show that we may find a nonseparating surface in a finite sheeted covering. We begin with a definition:

Definition. The size of a (possibly disconnected) surface $S$ is $\beta_{1}(S)$. We will say a surface $A$ is larger (resp. smaller) than a surface $B$ if $A$ has larger (resp. smaller) size.

We need:

Lemma 2.6. Suppose that $S$ is an oriented closed surface, not necessarily connected, embedded in the interior of an orientable 3-manifold $M$. Suppose that $S$ separates $M$ into two, not necessarily connected, submanifolds $M_{-}, M_{+}$, so that in both cases $\partial M_{ \pm}-S$ has size at least $2\left(\beta_{1}(S)+1\right)$. Then there is a 4 -fold cover $\tilde{M}$ of $M$ to which $S$ lifts, and so that we may choose two lifts $S_{1}$ and $S_{2}$ whose union does not separate $\tilde{M}$.

Proof. We claim that there are two properly embedded surfaces $A_{ \pm}$, one in each of $M_{ \pm}$representing nonzero primitive classes in $H_{2}\left(M_{ \pm}, \partial M_{ \pm}\right)$and both of which miss $S$. We temporarily defer the proof of the claim and prove the lemma: 
Let $\tilde{M}$ be the connected 4 -fold cover of $M$ constructed as follows. Consider the dual class to $A_{-} \cup A_{+}$lying in $H^{1}(M)$ and use this to define a homomorphism

$$
\pi_{1}(M) \longrightarrow \mathbf{Z}_{4} \text {. }
$$

It is clear from the construction that there are loops $\alpha_{ \pm} \in M_{ \pm}$which map to 2 in $\mathbf{Z}_{4}$. We form a single loop $\beta$ in $M$ as follows. Construct an arc $\gamma$ which runs from $\alpha_{-}$to $\alpha_{+}$. We may assume that $\gamma$ never hits any component of $S$ more than once. Using the arc, we construct $\beta$ in the obvious way so that it projects to $0 \in \mathbf{Z}_{4}$.

Let $S^{*}$ be a component of $S$ which meets $\gamma$, so that $\beta$ meets $S^{*}$ in precisely two points. The loop $\beta$ lifts, but neither $\alpha_{+}$nor $\alpha_{-}$does, this means we can find a lift $\tilde{\beta}_{1}$ which meets a pair of the pre-images of $S^{*}$ and another lift $\tilde{\beta}_{2}$ which only meets the disjoint pair. Choosing one pre-image from each of these pairs gives the required surfaces.

To prove the claim, observe that

$$
\operatorname{Ker}\left[H_{2}\left(M_{+}, \partial M_{+}\right) \longrightarrow H_{1}\left(\partial M_{+}\right) \longrightarrow H_{1}(S)\right]
$$

is nonzero since the rank of the domain is at least

$$
\frac{\beta_{1} \partial M_{+}}{2} \geq \beta_{1}(S)+1,
$$

which is greater than $\beta_{1}(S)$. Here the second map is given by projection using the direct sum decomposition of $H_{1}\left(\partial M_{+}\right)$coming from expressing

$$
\partial M_{+}=S \cup\left(\partial M_{+}-S\right) .
$$

Let $\alpha$ be a nontrivial element in the kernel; then $\alpha$ is represented by a properly embedded surface $A_{+}$in $M_{+}$. We can arrange that $A_{+}$meets the boundary of $M_{+}$ efficiently as follows.

By Poincaré duality, $\alpha$ is dual to a class in $H^{1}(M)$ and this gives an epimorphism of $\pi_{1} M$ onto $\mathbf{Z}$. One defines a simplicial map $f$ from $M$ to $S^{1}$ representing this map. Start with $f$ defined on $\partial M$ so that the pre-image $f^{-1} c$ of a certain point $c$ in the interior of some 1-simplex of $S^{1}$ is efficient. Then extend the map over $M$. Then $A_{+}$is the pre-image of $c$, which by transversality is a 2 -sided surface.

We have a surface $A_{+}$as required by the claim. Similarly one obtains $A_{-}$.

We recall that a property is said to be virtual if it holds for some subgroup of finite index.

Theorem 2.7. Let $M$ be a compact, connected irreducible 3-manifold with nonempty incompressible boundary all of whose components are tori. Suppose that the interior of $M$ has a complete hyperbolic structure of finite volume. Then $\pi_{1}(M)$ virtually maps onto a free group of rank 2 .

Proof. By passing to a finite cover $N$ of $M$ with at least 3 boundary components the proof of Theorem 2.3 finds a possibly disconnected, incompressible nonboundary parallel surface embedded in some $Y_{n}$. By choosing a $Y_{m}$ with $m$ much larger than $n$, we see that there is a finite cyclic covering $N_{m}$ of $N$ containing an embedding of an incompressible nonboundary parallel surface $S$ which separates $N_{m}$ into components each of which contains a number of boundary tori sufficient to apply Lemma 2.6 and find a covering of $N_{m}$ containing a pair of embedded disjoint incompressible surfaces whose union does not separate; this suffices to find the homomorphism onto a free group of rank 2. 
Corollary 2.8. Let $N$ be a compact, connected irreducible 3-manifold, with nonempty incompressible boundary, all of whose components are tori. Then either $N$ is covered by a product $T^{2} \times I$, or there is a finite cover of $N$ which contains an embedded nonseparating essential closed surface.

Proof. Take a torus decomposition for $N$. If this contains some nonboundary parallel torus, this may always be promoted to a nonseparating torus in a finite sheeted covering, for example by results contained in [4] or [6].

We assume then that the only tori in $N$ are boundary parallel, so that $N$ is either Seifert fibred or hyperbolic. The former case is easily dealt with by the observation that one can always create nonseparating vertical tori after passage to a finite sheeted covering except in the case when the base is a Euclidean orbifold. Since this orbifold has boundary, one deduces that $N$ is virtually a product.

The hyperbolic case is contained in the proof of Theorem 2.7.

The classes provided by Lemma 2.6 are nonperipheral and provide a degree one map from $M$ onto the wedge of two circles, so that the surfaces $S_{i}$ are pre-images of points in the interior of edges. By taking coverings of this graph and pulling back to coverings of $M$, we see we have shown:

Corollary 2.9. Let $M$ be a finite volume, noncompact hyperbolic 3-manifold. Then given any integer $K$, there is a finite sheeted covering $\tilde{M} \longrightarrow M$ so that $H_{2}(\tilde{M}) /$ incl $_{*}\left(H_{2}(\partial \tilde{M})\right)$ has rank at least $K$.

\section{Higher GENUS BOUNDARY}

This section is devoted to the proofs in the presence of higher genus boundary components. Some partial results in this direction were proved in [1].

Although the idea is very similar, the proof is complicated by the fact that the topology of the surface $G \subset Y_{n}$ does increase as $n$ increases; the strategy is to carefully arrange a partition of the boundary of some covering of $M$ so that the growth of the topology of $G$ is not too fast. To this end, we need:

Lemma 3.1. Suppose that $M$ is a compact irreducible 3-manifold with incompressible boundary and some boundary component has genus at least 2. Suppose also that $M$ is not an I-bundle over a surface. Then there is a finite cover $\tilde{M}$ of $M$ and a partition of the boundary of $\tilde{M}$ into two disjoint collections of components $A, B$ such that

$$
\beta_{1}(B)<\beta_{1}(A)<\beta_{1}(B)+(\# \text { compts of } A) .
$$

Proof. Let $C$ be any boundary component. Then we claim that there is a regular cover of $M$ with $C$ having at least 5 pre-images.

The reason is that the inclusion map of $\pi_{1}(C)$ into $\pi_{1}(M)$ must have image group of infinite index, else standard results imply that $M$ is an $I$-bundle. (See [5], Theorem 10.2.) Now [6] implies that $\pi_{1} C$ is subgroup separable in $\pi_{1}(M)$, so there is a homomorphism of $\pi_{1} M$ onto a finite group $G$ such that $\pi_{1} C$ maps to a subgroup $H$ of $G$ of index at least 5 . Thus to $C$ we may assign a subgroup $G(C)$ of $\pi_{1} M$, which contains $\pi_{1} C$ and has finite index at least 5 in $\pi_{1} M$. Let $G_{1}$ be the intersection over all boundary components $C$ of $G(C)$. Then $G_{1}$ has finite index in $G$. Let $G_{2}$ be the intersection of all conjugates of $G_{1}$; then $G_{2}$ is a normal subgroup of finite index in $G$. Let $N$ be the regular cover of $M$ corresponding to $G_{2}$. 
We recall that we have defined the notion of size and note that we have arranged that every component of $\partial M$ has at least 5 pre-images, and since the cover is regular each element in this set of pre-images has the same size.

We will now rename $N$ as $M$. Thus we may assume that whenever $M$ has a boundary component, there are at least 5 boundary components of the same size. Partition the boundary of $M$ into 3 sets of components $A, B, C$ in such a way that for every size $n$, the number of components of size $n$ in each of these sets differs by at most one from each other. In other words, if $X$ is a set of surfaces we define $\#_{n}(X)$ to be the number of connected surfaces in $X$ with size $n$. Then for all $n$, the numbers $\#_{n}(A), \#_{n}(B), \#_{n}(C)$ differ by at most 1 . We will assume that $C$ is at least as large as $A$ and $B$.

The size of $A \cup B$ is greater than the size of $C$; therefore, using the argument in 2.2 there is an efficient surface $F$ in $M$ with nonempty boundary contained in $A \cup B$. Let

$$
\pi: M_{F, p} \longrightarrow M
$$

be the $p$-fold cyclic cover of $M$ dual to $F$ where $p$ is a number much larger than the size of the boundary of $M$. Denote by $\tilde{A}, \tilde{B}, \tilde{C}$ the pre-images of $A, B, C$ under this covering. Since $F$ is disjoint from $C$, every component of $\pi^{-1} C$ maps injectively under the covering. Thus $\tilde{C}$ is at least as large as $\tilde{A}$ and $\tilde{B}$. Thus $\tilde{C}$ has at least $p$ components, and $p$ is much larger than the size of each of these components. We may create two new sets $\tilde{A}^{+}, \tilde{B}^{+}$by taking the sets $\tilde{A}, \tilde{B}$ and enlarging them by sharing the components of $\tilde{C}$ between them. We wish to do this so that the sizes of $\tilde{A}^{+}, \tilde{B}^{+}$differ by at most the size of the largest component of $\tilde{C}$. This is possible because the size of $\tilde{C}$ exceeds the difference in the sizes of $\tilde{A}$ and $\tilde{B}$. The resulting collection of surfaces $\tilde{A}^{+}, \tilde{B}^{+}$have of order $p$ components each. They differ in size by at most the size of the largest component of $\tilde{C}$ which equals the size of the largest component of $C$. But this is less than the size of the boundary of $M$ which is very small compared to $p$. We may assume that $\tilde{B}^{+}$is strictly smaller than $\tilde{A}^{+}$, and since the number of components of $\tilde{A}^{+}$is much larger than the difference in the size of these two surfaces,

$$
\operatorname{size}\left(\tilde{B}^{+}\right)<\operatorname{size}\left(\tilde{A}^{+}\right)<\operatorname{size}\left(\tilde{B}^{+}\right)+\left(\# \text { compts of } \tilde{A}^{+}\right) .
$$

Lemma 3.2. Suppose that the compact 3-manifold $M$ has boundary partitioned into sets $A$ and $B$ such that

$$
\beta_{1}(B)<\beta_{1}(A)<\beta_{1}(B)+(\# \text { compts of } A) .
$$

Then there is a finite covering of $M$ which contains an embedded nonseparating closed incompressible surface.

Proof. Since $\partial M=A \cup B$ and $A$ is larger, there is an efficient surface $F$ properly embedded in $M$ with nonempty boundary contained in $A$. Take any components of $A$ which are disjoint from $F$ and remove them from $A$ and put then into $B$. This makes $B$ larger and $A$ smaller, thus we still have that

$$
\beta_{1}(A)<\beta_{1}(B)+(\# \text { compts of } A) \text {. }
$$

The idea is the following. One proceeds as in the case that the boundary consists of tori. However the surface $G$ is no longer the double of $F$ since $A$ is covered by high genus surfaces. Taking an $n$-fold cyclic cover multiplies the Euler characteristic by 
$n$. Thus although $A$ is slightly larger than $B$ the fact that it has many components and these all unwrap in the cover means that the size of the $n$-fold cover of $A$ is, approximately,

$$
n \cdot[\operatorname{size}(A)-2(\# \text { compts of } A)] \text {. }
$$

But the size of the $n$-fold cover of $B$ is $n$ times the size of $B$ since $B$ lifts. Thus for $n$ large, the pre-image of $B$ is larger than that of $A$.

Now the details. Let

$$
\pi: M_{F} \longrightarrow M
$$

be the infinite cyclic cover of $M$ dual to $F$. The submanifold $X$ of $M$ is obtained by cutting $M$ open along $F$, thus $X \cong M-F \times(-1,1)$ and $\partial X=F_{+} \cup F_{-} \cup A^{-}$ where $A^{-}$is $A$ cut open along $F \cap A$. Then $M_{F} \cong \bigcup_{i} X_{i}$ where $X_{i}$ is a copy of $X$ and these copies are glued together along $F_{ \pm}=F \times \pm 1$. Let $Y_{n}=\bigcup_{i=1}^{n} X_{i}$ and partition the boundary of $Y_{n}$ as $Y_{n}=G \cup W$ where $W=\pi^{-1} B$ and $G=\partial Y_{n}-W$ is the rest. We repeat the analysis done before, the only important difference is that this time $G$ is not the double of $F$ since the covering $\pi^{-1} A$ of $A$ is not a collection of annuli, but has more complicated topology.

We claim that $G$ is connected. This is because each component of $\pi^{-1} A$ is noncompact and therefore meets both $\tilde{F}_{0}, \tilde{F}_{n}$. But $G$ is the union of $\tilde{F}_{0}, \tilde{F}_{n}$ together with the subsurface of $\pi^{-1} A$ lying in $Y_{n}$, and so every component of $Y_{n} \cap \pi^{-1} A$ has boundary which is in one or both of $\tilde{F}_{0}, \tilde{F}_{n}$. Furthermore, some component of $Y_{n} \cap \pi^{-1} A$ meets both $\tilde{F}_{0}, \tilde{F}_{n}$.

Noting that gluing bounded surfaces along circles does not change the Euler characteristic, we now compute the Euler characteristic of $G$ as $\chi_{G}=2 \chi_{F}+n \chi_{A_{-}}$ where the surface $A_{-}$is obtained by deleting annuli from the original surface $A \subset$ $\partial M$, so that $\chi_{A_{-}}=\chi_{A}$. Since $G$ is connected we obtain:

$$
\chi_{G}=2-\beta_{1}(G)=2 \chi_{F}+n \chi_{A}=2 \chi_{F}+n\left(2(\text { \#compts of } A)-\beta_{1}(A)\right)
$$

so that

$$
\beta_{1}(G)=2-2 \chi_{F}+n\left(\beta_{1}(A)-2(\# \text { compts of } A)\right) .
$$

However every component of $B$ lifts, so that $\beta_{1}(W)=n \cdot \beta_{1}(B)$ and a comparison of growth rates provided by the hypothesis completes the proof as in the torus case:

For $G$ is homologous to $W$ in $Y_{n}$ so that if compression of $G$ results in an incompressible surface $S$ consisting only of spheres and components parallel to components of $W$, then

$$
\beta_{1}(W) \leq \beta_{1}(S) \leq \beta_{1}(G) .
$$

But the inequality of the hypothesis together with the remarks of the above paragraph then give:

$$
n \cdot \beta_{1}(B) \leq 2-2 \chi_{F}+n\left(\beta_{1}(A)-2(\# \text { compts of } A)\right)<2-2 \chi_{F}+n\left(\beta_{1}(B)-2\right),
$$

but this is a contradiction for large $n$.

Thus one obtains an incompressible surface $S$, not necessarily connected, which separates $W$ from the rest of $\partial M_{F}$. Again, using Lemma 2.6, one argues that there is some finite cover with a component of the pre-image of $S$ nonseparating.

Putting together Lemma 3.1 and Lemma 3.2 we have shown:

Theorem 3.3. Let $M$ be a compact, connected 3-manifold with nonempty incompressible boundary. Suppose that the interior of $M$ admits a complete hyperbolic structure. Then either $M$ is covered by a product $F \times I$, or $M$ contains an essential surface $S$ of genus at least 2. 
Furthermore $S$ can be lifted to an embedded nonseparating surface in a finite cover of $M$.

Exactly as in 2.4 we deduce:

Corollary 3.4. Let $M$ be a compact, connected irreducible manifold with nonempty incompressible boundary. Then either $M$ is covered by a product $F \times I$, or $M$ contains an essential closed surface.

To deduce the results concerning virtually freeness and increasing nonperipheral homology stated in the Introduction, we note in the proof of Lemma 3.2 that we showed, for very large $n$, the surface $G$ has much smaller size than the rest of the boundary in $Y_{n}$, so that we may apply Lemma 2.6. Thus we have shown

Theorem 3.5. Let $M$ be a compact, connected 3-manifold with nonempty incompressible boundary. Suppose that the interior of $M$ admits a complete hyperbolic structure. Then either $M$ is covered by a product $T^{2} \times I$, or $\pi_{1}(M)$ virtually maps onto a free group of rank 2.

Observe that if $F$ is a closed surface of genus at least $2, \pi_{1}(F)$ surjects the free group of rank 2 , so that the exclusion of products is unnecessary except for the case $T^{2} \times I$.

Corollary 3.6. Let $M$ be a compact, connected irreducible 3-manifold with nonempty incompressible boundary. Then either $M$ is covered by a product $F^{2} \times I$, or, given any integer $K$, there is a finite sheeted covering $\tilde{M} \longrightarrow M$ so that $H_{2}(\tilde{M}) / \operatorname{incl}_{*}\left(H_{2}(\partial \tilde{M})\right)$ has rank at least $K$.

Remark. The existence of a map to the free group of rank 2 in the higher genus boundary case is a logical deduction from the case where all boundary components are tori, as we discuss briefly below. However, the additional feature of the proof of Theorem 3.5 and Corollary 3.6 is that the essential surfaces are closed and the homology is nonperipheral.

To see the deduction, assume that $M$ has a boundary component $Y$ of genus $g>1$. Using [7] we can perform a sequence of 2-handle attachments to $Y$ so that the result is hyperbolic with $Y$ replaced by a disjoint union of some number of tori. We can repeat this for every such boundary component and then apply Theorem 2.7 .

Theorem 3.7. Let $M$ be an irreducible 3-manifold with nonempty incompressible boundary. Then either $M$ is covered by a product $T^{2} \times I$, or $\pi_{1}(M)$ virtually maps onto a free group of rank 2 .

Proof. The proof is similar to that of Corollary 2.8. If the torus decomposition of $M$ contains a nonperipheral torus, we apply [6]. If all tori are peripheral, then either $M$ is Seifert fibred or hyperbolic.

In the former case, we virtually get a map onto free group of rank 2 unless the base is a Euclidean orbifold. The hyperbolic case is covered by Theorem 3.5.

\section{REFERENCES}

[1] J. W. Anderson, Closed essential surfaces in hyperbolizable acylindrical manifolds. Preprint.

[2] B. Freedman \& M.H. Freedman Haken finiteness for bounded 3-manifolds, locally free groups and cyclic covers. Preprint. 
[3] D. Gabai On 3-manifolds finitely covered by surface bundles, In Low-dimensional Topology and Kleinian Groups, L. M. S. Lecture Note Series, vol 112, Ed. D. B. A. Epstein, pp 146-155, C. U. P. (1986). MR 88m:57021

[4] J. Hempel. Residual finiteness for 3-Manifolds. In Combinatorial Group Theory and Topology. Ann. of Math. Studies, vol. 111 pp. 379 -396, P. U. P (1987). MR 89b:57002

[5] J. Hempel. 3-manifolds. Ann. of Math. Studies, vol. 86, P. U. P. (1976). MR 54:3702

[6] D.D. Long \& G. Niblo. Subgroup Separability and 3-manifold groups. Math. Zeit. vol. 207 (1991), pp.209 - 215. MR 92g:20047

[7] M. Scharlemann \& Y. Q. Wu Hyperbolic manifolds and degenerating handle additions. J. Aust. Math. Soc. vol. 55 (1993) pp. 72 -89. MR 94e:57019

[8] W.P. Thurston. The Geometry and Topology of 3-manifolds. Princeton University mimeographed notes. (1979)

[9] W.P. Thurston. Three dimensional manifolds, Kleinian groups and hyperbolic geometry. Bull. A.M.S. vol 6 (1982) pp. 357 - 381. MR 83h:57019

[10] F. Waldhausen. On irreducible three-manifolds which are sufficiently large. Ann. of Math. 87 (1968) pp. 56 - 68. MR 36:7146

(D. Cooper and D. D. Long) Department of Mathematics, University Of California, Santa Barbara, California 93106

(A. W. Reid) Department of Mathematics, University of Texas, Austin, Texas 78712

E-mail address: areid@math.utexas.edu 